\title{
Propiedades psicométricas del inventario de estilos de aprendizaje de Kolb y del cuestionario de Felder-Silverman en estudiantes de educación física de Santiago de Chile
}

\author{
Psychometric properties of the inventory of learning stylez of Kolb and Felder- \\ Silverman questionnaire in student of physical education of Santiago of Chile
}

*Fernando Maureira Cid, **Elizabeth Flores Ferro, *Patricia González Flores,

*Elvira Palma Gajardo, *Miguel Fernández Rebolledo, ***Carlos Véliz Véliz

Maureira, F., Flores, E., González, P., Palma, E., Fernandez, M., \& Véliz, C. (2018). Propiedades psicométricas del inventario de estilos de aprendizaje de Kolb y del cuestionario de Felder-Silverman en estudiantes de educación física de Santiago de Chile. Revista Ciencias de la Actividad Física UCM, N 19(1) enero-junio, 1-11. DOI: http://doi.org/10.29035/rcaf.19.1.5

\begin{abstract}
RESUMEN
El objetivo del presente estudio fue conocer las propiedades psicométricas del Inventario de Estilos de Aprendizaje de Kolb y del Cuestionario de Felder-Silverman en una muestra de estudiantes de educación física de Chile. Para ello se aplicó ambos instrumentos a 141 estudiantes de dicha carrera de la Universidad Católica Silva Henríquez, siendo el 34,8\% mujeres y el 65,2\% hombres. Los resultados muestran que las cuatro sub-escalas del inventario de Kolb y las 4 sub-escalas del cuestionario de Felder-Silverman poseen índices adecuados de validez, sin embargo, los índices de confiabilidad obtenidos con alfa de Cronbach se ubican bajo los niveles aceptables en todas las sub-escalas. Se recomiendan más estudios con muestras de diversas universidades para confirmar las propiedades psicométricas de estos instrumentos en este tipo de población.
\end{abstract}

\section{PALABRAS CLAVE}

Estilos de aprendizaje, educación física, modelo de Kolb, modelo de Felder-Silverman, estudiantes universitarios, propiedades psicométricas.

\begin{abstract}
The aim of the present study was to know the psychometric properties of the learning styles inventory of Kolb and Felder-Silverman's questionnaire in a students' sample of physical education of Chile. For it both instruments were applied to 141 students of the above mentioned career of the Universidad Católica Silva Henríquez, being 34,8\% ladies and 65,2 \% males. The results show that four sub-scales of Kolb's inventory and 4 sub-scales of Felder-Silverman's questionnaire possess suitable indexes of validity, nevertheless, the indexes of reliability obtained with Cronbach's alpha are located under the acceptable levels in all the sub-scales. More studies are recommended by samples of diverse universities to confirm the psychometric properties of these instruments in this type of population.
\end{abstract}

\section{Key words}

Learning styles, physical education, model of Kolb, model of Felder-Silverman, university students, psychometric properties.

\footnotetext{
* Escuela de Educación en Ciencias del Movimiento y Deportes. Universidad Católica Silva Henríquez. Santiago de Chile.

** Estudiante Doctorado en Educación. Universidad SEK. Santiago de Chile.

*** Entrenador nado sincronizado. Gimnasio Pulse, Estadio Mayor. Santiago de Chile.
} 


\section{INTRODUCCIÓN}

Los estilos de aprendizaje hacen referencia al modo preferido de cada individuo para aprender nueva información (Dunn y Dunn, 1978), siendo definidos como los rasgos cognitivos, afectivos y fisiológicos que constituyen indicadores estables de la forma como aprende una persona (Keefe, 1988). En la actualidad existen muchos modelos que tratan de explicar cómo percibimos y procesamos la información, destacando el de Dunn y Dunn (1974), Kolb (1984), Felder y Silverman (1988), Herrmann (1990), Alonso, Gallego y Honey (1994), etc.

El modelo de Kolb (1984) se basa en un ciclo de cuatro etapas: a) experiencia concreta, que involucra relacionarse con las personas y las situaciones diarias, aprendiendo a través de las relaciones y los sentimientos; b) observación reflexiva, que involucra pensar las situaciones desde diversos puntos de vista, buscando significado a las cosas y aprendiendo a través de la observación; c) conceptualización abstracta, que involucra el pensamiento lógico, la planificación y el entendimiento secuencial de los problemas; d) experimentación activa, que involucra el hacer, experimentar, vivenciar y aprender a través del cambio de situaciones y de la acción.

A partir de estas etapas Kolb (1984) identifica cuatro tipos de aprendedores:

a) Acomodador, que surge de la experiencia concreta y la experimentación activa. Se caracterizan por aprender de la experiencia, ser pragmáticos, adaptarse fácilmente, gustan de las actividades en grupo, resolver problemas en forma intuitiva, buscar información en otros, tomar riesgos, etc.

b) Divergente, que surge de la experiencia concreta y la observación reflexiva. Se caracterizan por su imaginación, por aplicar más la observación que la acción, son buenos para identificar problemas y compartir información, son sensitivos, se interesan en el arte, consideran las situaciones desde diversas perspectivas, etc. c) Asimilador, que surge de la observación reflexiva y la conceptualización abstracta. Se caracterizan por la capacidad de crear modelos teóricos, por razonamiento inductivo, gustan de resolver problemas y crear experimentos, resuelven los problemas leyendo y reflexionando, gustan de las ideas y conceptos abstractos, etc.

d) Convergente, que surge de la conceptualización abstracta y la experimentación activa. Se caracterizan por su capacidad para resolver problemas, son prácticos, gustan de las actividades donde solo hay una solución, poseen buen razonamiento hipotético-deductivo, buscan el uso práctico a las ideas, gustan poco de las personas, etc.

Un estudio de Escurra (1992) mostró que el Inventario de Estilos de Aprendizaje (IEA) de Kolb posee dos factores: concreto-abstracta y actividad-reflexión, que explican el 52,48\% de la varianza, además el análisis de traslapamiento y el análisis paralelo indican como válidos los dos factores del instrumento. También presenta valores de alfa de Cronbach entre 0,67 y 0,87 , lo que muestra la confiabilidad del inventario. Por su parte, Delgado (2004) encontró valores de correlación ítem-test superiores a 0,20 en cada uno de los conceptos del IEA de Kolb y valores de confiabilidad, mediante alfa de Cronbach, entre 0,696 y 0,712. Otros trabajos como Hudak y Anderson (1990), Willcoxson y Prosser (1996), Castaño (2005), Kayes (2005), etc., muestran valores adecuados de confiabilidad y validez del inventario de Kolb.

Por su parte, el modelo de Felder y Silverman (1988) se basa en estilos de aprendizaje con cuatro dimensiones bipolares: activo/ reflexivo; sensitivo/intuitivo; visual/verbal y secuencial/global. Estos perfiles tienen las siguientes características:

a) Activos: son estudiantes que aplican conocimientos, prueban las cosas para saber cómo funcionan, comprenden mejor la nueva información cuando la discuten y la aplican, les gusta trabajar en grupos, aprenden mejor haciendo. 
b) Reflexivos: son estudiosos; sujetos racionales, lógicos, que gustan de trabajar solos, metódicos y ordenados, aprenden mejor pensando.

c) Sensitivos: son estudiantes que gustan de problemas con soluciones claras y establecidas, son prácticos y cuidadosos, su aprendizaje se basa en hechos, no les gustan las sorpresas.

d) Intuitivos: son estudiantes que gustan de la innovación, establecer relaciones, son rápidos para trabajar, gustan de los procesos que requieran mucha memorización.

e) Visuales: son estudiantes que aprenden mejor con didácticas gráficas, películas, presentaciones, etc.

f) Verbales: son estudiantes que aprenden mejor con explicaciones verbales y escritas.

g) Secuenciales: son estudiantes que buscan soluciones mediante pasos lógicos.

h) Globales: son estudiantes que aprenden sin seguir un orden, pero entienden fácilmente el sentido global de un conjunto de información, resuelven problemas en forma novedosa.

Un estudio de Brito y Espinosa (2014) con 459 estudiantes de medicina de la Universidad Autónoma de San Luis Potosí, México, mostró índices de fiabilidad entre 0,38 y 0,61 en el cuestionario de estilos de aprendizaje de Felder y Silverman. Además, los estudiantes mostraron preferencias por el estilo activo, sensitivo, visual y secuencial. Por su parte, Puello, Fernández y Cabarcas (2014) evaluaron a estudiantes de ingeniería de sistemas de la Universidad de Cartagena, Colombia. Los resultados muestran que el $77 \%$ presenta un perfil activo, el $73 \%$ es sensitivo, el $82 \%$ es visual y el $68 \%$ es secuencial.

Un trabajo de Hosford y Siders (2010), donde evaluaron a 340 estudiantes de diferentes carreras de la Greek university en Grecia, entregó valores de alfa de Cronbach aceptables para cada dimensión del cuestionario de Fel- der y Silverman, ( $\alpha=0,63$ sub-escala activo/ reflexivo; $\alpha=0,76$ sub-escala sensitivo/intuitivo; $\alpha=0,64$ sub-escala visual/verbal y $\alpha=0,62$ sub-escala secuencial/global). Otro estudio sobre las propiedades psicométricas del cuestionario de Felder y Silverman fue realizado por Al-Azawei, Parslow y Lundqvist (2015) con 259 estudiantes de ingeniería de la University of Babylon de Iraq, entregando la existencia de 17 factores que explicaban el 61,91\% de la varianza, con correlaciones bajas y positivas entre las 4 sub-escalas (entre $\mathrm{r}=0,148$ y $\mathrm{r}=$ $0,414)$. Finalmente, el estudio entregó valores de confiabilidad de $\alpha=0,41$ sub-escala activo/ reflexivo; $\alpha=0,57$ sub-escala sensitivo/intuitivo; $\alpha=0,59$ sub-escala visual/verbal y $\alpha=0,43$ sub-escala secuencial/global.

En nuestro país existen estudios que muestran las propiedades psicométricas de instrumentos para evaluar los estilos de aprendizaje en estudiantes de educación física (Flores y Maureira, 2015; Maureira, 2013; Maureira, 2015), pero no se encontraron trabajos que muestren los índices de validez y confiabilidad del Inventario de Kolb y del cuestionario de Felder-Silverman, debido a eso es que surge el objetivo de la presente investigación: conocer las propiedades psicométricas de ambos instrumentos en una muestra de estudiantes de educación física de Santiago de Chile.

\section{MATERIAL Y MÉTODO}

Muestra: Se trabajó con una muestra no probabilística intencional compuesta por 141 estudiantes de educación física de la Universidad Católica Silva Henríquez de la ciudad de Santiago de Chile. Del total, 49 estudiantes son mujeres $(34,8 \%)$ y 92 son hombres $(65,2 \%)$. Con respecto al nivel cursado dentro de la carrera, 56 estudiantes cursan primer año (39,7\%), 59 cursan tercer año (41,8\%) y 26 cursan cuarto año (18,5\%). La edad mínima fue 17 años y la máxima de 28 años con una media de 21,0 2,40. El inventario de Kolb fue constatado correctamente por 122 estudiantes y el cuestionario de Felder-Silverman por 139 integrantes de la muestra. 
Instrumento: Se utilizó el Inventario de Estilos de Aprendizaje de Kolb (1984) que consta de 36 adjetivos que describen tipos de conductas organizadas en nueve columnas con 4 conceptos cada uno. El evaluado debe calificar los adjetivos de cada columna con valores entre $1=$ menos dominante y $4=$ más dominante. Los resultados entregan una clasificación entre cuatro estilos posibles: acomodador, divergente, asimilador o convergente.

También se utilizó el Inventario de estilos de aprendizaje de Felder-Silverman (1988) que consta de 44 preguntas con dos alternativas de respuestas. Este instrumento consta de cuatro dimensiones bipolares: activo-reflexivo, sensitivo-intuitivo, visual-verbal y secuencial-global. Puntajes entre $-3 y+3$ en cada dimensión sugieren un estilo de aprendizaje equilibrado entre los dos polos de la escala, puntajes entre -7 y -5 indican preferencia moderada hacia el estilo activo, sensitivo, visual o secuencial, puntajes entre $+5 y+7$ indican preferencia moderada hacia el estilo reflexivo, intuitivo, verbal o global, finalmente, puntajes entre -11 y -9 indican una fuerte preferencia hacia el estilo activo, sensitivo, visual o secuencial, y puntajes entre $+9 y+11$ indican fuerte preferencia hacia el estilo reflexivo, intuitivo, verbal o global.

Procedimiento: La recolección de datos tuvo una duración 15 a 20 minutos aproximadamente y fue realizada en forma grupal durante las horas de clases. Cada estudiante tuvo la opción de participar o no en la investigación si lo deseaba. Cada estudiante que conformó la muestra firmó un consentimiento informado.

Análisis de datos: Se utilizó el programa estadístico SPSS 22.0 para Windows. Para los análisis de confiabilidad y validez del Inventario de Kolb y del cuestionario de Felder-Silverman se aplicaron análisis factoriales exploratorios con rotaciones Varimax para determinar la validez de constructo de cada sub-escala de ambos instrumentos y pruebas de alfa de Cronbach para los niveles de confiabilidad de los test.

\section{RESULTADOS}

\section{Propiedades psicométricas del inventario de Kolb}

No se realizaron cambios semánticos en los ítems, ya que no hubo confusión ni problemas con su interpretación por parte de los estudiantes de educación física. La sub-escala Experiencia Concreta presenta un KMO de 0,530 y una prueba de esfericidad de Bartlett de $\mathrm{X}^{2}=28,493 ; \mathrm{gl}=15 ; \mathrm{p}=0,019$ por lo que se procedió a realizar un análisis factorial de componentes principales y rotación de Varimax. Este reveló la existencia de 3 componentes que explican el $61,4 \%$ de la varianza (Tabla 1). Todos los ítems saturaron sobre 0,600 en algún factor. Esta sub-escala obtuvo un alfa de Cronbach de 0,223.

\section{Tabla 1}

Análisis factorial de los componentes principales después de rotación Varimax de la sub-escala Experiencia Concreta.

\begin{tabular}{lccc}
\hline Concepto & Factor 1 & Factor 2 & Factor 3 \\
\hline Sintiendo & 0,738 & & \\
\hline Intuitivamente & 0,700 & & \\
\hline Viviendo situaciones & 0,630 & & \\
\hline Receptivamente & & 0,777 & \\
\hline Orientándome al presente & 0,761 & \\
\hline Aceptando & & 0,926 \\
\hline \% varianza & 20,335 & 17,203 \\
\hline$\%$ varianza total & & 61,449 \\
\hline
\end{tabular}

Factor 1 = aprendizaje por experiencia; Factor 2 = aprendizaje presente; Factor 3 = aceptación 
La sub-escala Observación Reflexiva presenta un KMO de 0,633 y una prueba de esfericidad de Bartlett de $\mathrm{X}^{2}=49,303 ; \mathrm{gl}=15 ; \mathrm{p}=$ 0,000. El análisis factorial reveló la existencia de 2 componentes que explican el $48,5 \%$ de la varianza (Tabla 2). Todos los ítems saturaron sobre 0,400 en algún factor. Esta sub-escala obtuvo un alfa de Cronbach de 0,410 .

Tabla 2

Análisis factorial de los componentes principales después de rotación Varimax de la sub-escala Observación Reflexiva.

\begin{tabular}{lccc}
\hline Concepto & Factor 1 & Factor 2 & Factor 3 \\
\hline Observando atentamente & 0,687 & & \\
\hline Observando detalles & 0,742 & & \\
\hline Observando & 0,747 & & \\
\hline Ensayando & & 0,419 & \\
\hline Reflexivamente & 0,647 & \\
\hline Reservadamente & 0,765 & 0,926 \\
\hline \% varianza & 28,786 & 19,712 & 17,203 \\
\hline \% varianza total & & 48,499 & 61,449 \\
\hline
\end{tabular}

Factor 1 = observación cautelosa

Factor 2 = observación reflexiva

La sub-escala Conceptualización Abstracta presenta un KMO de 0,511 y una prueba de esfericidad de Bartlett de X2 = 24,760; $\mathrm{gl}=15 ; \mathrm{p}$ $=0,049$. El análisis factorial reveló la existencia de 3 componentes que explican el $60,7 \%$ de la varianza (Tabla 3 ). Todos los ítems saturaron sobre 0,600 en algún factor. Esta sub-escala obtuvo un alfa de Cronbach de 0,295.

Tabla 3

Análisis factorial de los componentes principales después de rotación Varimax de la sub-escala Conceptualización Abstracta.

\begin{tabular}{lccc}
\hline Concepto & Factor 1 & Factor 2 & Factor 3 \\
\hline Pensando & 0,626 & & \\
\hline Cuidadosamente & 0,606 & & \\
\hline Conceptualizando & 0,720 & & \\
\hline Lógicamente & & 0,719 & \\
\hline Racionalmente & 0,728 & \\
\hline Analíticamente & & 0,888 \\
\hline \% varianza & & & 18,445 \\
\hline \% varianza total & 21,466 & & 60,766 \\
\hline
\end{tabular}

Factor 1 = definiendo contenidos

Factor 2 = razonando

Factor 3 = examinando 
La sub-escala Experimentación Activa presenta un KMO de 0,650 y una prueba de esfericidad de Bartlett de $\mathrm{X} 2=55,844 ; \mathrm{gl}=15 ; \mathrm{p}=$ 0,000. El análisis factorial reveló la existencia de 3 componentes que explican el $64,7 \%$ de la varianza (Tabla 4). Todos los ítems saturaron sobre 0,500 en algún factor. Esta sub-escala obtuvo un alfa de Cronbach de 0,418.

\section{Tabla 4}

Análisis factorial de los componentes principales después de rotación Varimax de la sub-escala Experimentación Activa.

\begin{tabular}{lccc}
\hline Concepto & Factor 1 & Factor 2 & Factor 3 \\
\hline Practicando & 0,713 & & \\
\hline Haciendo & 0,757 & & \\
\hline Activamente & 0,681 & & \\
\hline Pragmáticamente & 0,515 & & \\
\hline Responsablemente & & 0,906 & \\
\hline Probar ideas & & 0,973 \\
\hline \% varianza & 30,214 & 17,605 & 16,944 \\
\hline \% varianza total & & & 64,763 \\
\hline
\end{tabular}

Factor 1 = realizando

Factor 2 = responsable

Factor 3 = probando ideas

En la Tabla 5 se observan las correlaciones entre las diferentes sub-escalas del Inventario de Kolb. Es posible observar que la Experiencia Concreta se relaciona en forma negativa con la Observación Reflexiva $\left(\mathrm{r}=-0,205 ; \mathrm{r}^{2}=0,04\right) \mathrm{y}$ la Conceptualización Abstracta $\left(\mathrm{r}=-0,601 ; \mathrm{r}^{2}=\right.$
0,36). De igual forma, la Observación Reflexiva y la Experimentación Activa se relacionan negativamente $\left(\mathrm{r}=-0,476 ; \mathrm{r}^{2}=0,23\right)$ y la Conceptualización Abstracta y la Experimentación Activa $\left(\mathrm{r}=-0,268 ; \mathrm{r}^{2}=0,07\right)$.

\section{Tabla 5}

Correlaciones entre las diferentes sub-escalas del inventario de Kolb.

\begin{tabular}{lccc}
\hline & EC & OR & CA \\
\hline Experimentación Concreta (EC) & & & \\
\hline Observación Reflexiva (OR) & $-0,205^{\star}$ & & \\
\hline Conceptualización Abstracta (CA) & $-0,601^{\star *}$ & 0,121 & \\
\hline Experimentación Activa (EA) & $-0,040$ & $-0,479^{\star *}$ & $-0,268^{\star *}$ \\
\hline
\end{tabular}

* correlación significativa al nivel 0,05

** correlación significativa al nivel 0,01 


\section{Propiedades psicométricas del cuestionario de Felder-Silverman}

No se realizaron cambios semánticos en los ítems, ya que no hubo confusión ni problemas con su interpretación por parte de los estudiantes de educación física. La sub-escala Activo-Reflexivo presenta un KMO de 0,578 y una prueba de esfericidad de Bartlett de $\mathrm{X} 2=$ 105,$650 ; \mathrm{gl}=55 ; \mathrm{p}=0,000$ por lo que se pro-

cedió a realizar un análisis factorial de componentes principales y rotación de Varimax. Esto reveló la existencia de 4 componentes que explican el 52,2\% de la varianza (Tabla 6). Todos los ítems saturaron sobre 0,370 en algún factor. Esta sub-escala obtuvo un alfa de Cronbach de 0,475 .

\section{Tabla 6}

Análisis factorial de los componentes principales después de rotación Varimax de la sub-escala Activo-Reflexivo.

\begin{tabular}{|c|c|c|c|c|}
\hline & Factor 1 & Factor 2 & Factor 3 & Factor 4 \\
\hline Pregunta 1 & 0,624 & & & \\
\hline Pregunta 37 & 0,481 & & & \\
\hline Pregunta 41 & 0,741 & & & \\
\hline Pregunta 13 & & 0,669 & & \\
\hline Pregunta 17 & & 0,636 & & \\
\hline Pregunta 21 & & 0,521 & & \\
\hline Pregunta 5 & & & 0,744 & \\
\hline Pregunta 9 & & & 0,541 & \\
\hline Pregunta 33 & & & 0,374 & \\
\hline Pregunta 25 & & & & 0,785 \\
\hline Pregunta 29 & & & & 0,736 \\
\hline$\%$ varianza & 14,180 & 13,430 & 12,941 & 11,648 \\
\hline$\%$ varianza total & & & & 52,199 \\
\hline
\end{tabular}

Factor 1 = aprendizaje por experiencia

Factor 2 = aprendizaje presente

Factor 3 = aceptación

La sub-escala Sensitivo-Intuitivo presenta un KMO de 0,504 y una prueba de esfericidad de Bartlett de X2 = 105,188; gl = 55; p = 0,000. El análisis factorial reveló la existencia de 5 com- ponentes que explican el 60,6\% de la varianza (Tabla 7). Todos los ítems saturaron sobre 0,400 en algún factor. Esta sub-escala obtuvo un alfa de Cronbach de 0,325. 
Tabla 7

Análisis factorial de los componentes principales después de rotación Varimax de la sub-escala Sensitivo-Intuitivo.

\begin{tabular}{|c|c|c|c|c|c|}
\hline & Factor 1 & Factor 2 & Factor 3 & Factor 4 & Factor 5 \\
\hline Pregunta 2 & 0,768 & & & & \\
\hline Pregunta 18 & $-0,602$ & & & & \\
\hline Pregunta 22 & 0,446 & & & & \\
\hline Pregunta 30 & 0,497 & & & & \\
\hline Pregunta 6 & & 0,827 & & & \\
\hline Pregunta 38 & & 0,695 & & & \\
\hline Pregunta 14 & & & 0,700 & & \\
\hline Pregunta 42 & & & 0,743 & & \\
\hline Pregunta 26 & & & & 0,867 & \\
\hline Pregunta 10 & & & & & $-0,741$ \\
\hline Pregunta 34 & & & & & 0,581 \\
\hline$\%$ varianza & 14,259 & 13,118 & 11,837 & 10,922 & 10,470 \\
\hline$\%$ varianza total & & & & & 60,607 \\
\hline
\end{tabular}

Factor 1 = aprendizaje por experiencia; Factor 2 = aprendizaje presente; Factor 3 = aceptación

La sub-escala Visual-Verbal presenta un KMO de 0,633 y una prueba de esfericidad de Bartlett de $\mathrm{X} 2=119,976 ; \mathrm{gl}=55 ; \mathrm{p}=0,000$. El análisis factorial reveló la existencia de 4 componentes que explican el 52,9\% de la varianza (Tabla 8 ). Todos los ítems saturaron sobre 0,500 en algún factor. Esta sub-escala obtuvo un alfa de Cronbach de 0,557.

\section{Tabla 8}

Análisis factorial de los componentes principales después de rotación Varimax de la sub-escala Visual-Verbal.

\begin{tabular}{|c|c|c|c|c|}
\hline & Factor 1 & Factor 2 & Factor 3 & Factor 4 \\
\hline Pregunta 7 & 0,673 & & & \\
\hline Pregunta 11 & 0,583 & & & \\
\hline Pregunta 15 & 0,658 & & & \\
\hline Pregunta 31 & 0,638 & & & \\
\hline Pregunta 3 & & 0,514 & & \\
\hline Pregunta 35 & & 0,523 & & \\
\hline Pregunta 39 & & 0,758 & & \\
\hline Pregunta 23 & & & 0,576 & \\
\hline Pregunta 43 & & & 0,726 & \\
\hline Pregunta 19 & & & & 0,780 \\
\hline Pregunta 27 & & & & 0,742 \\
\hline$\%$ varianza & 16,218 & 12,464 & 12,134 & 12,125 \\
\hline$\%$ varianza total & & & & 52,940 \\
\hline
\end{tabular}

Factor 1 = definiendo contenidos; Factor 2 = razonando; Factor 3 = examinando. 
La sub-escala Secuencial-Global presenta un KMO de 0,556 y una prueba de esfericidad de Bartlett de X2 = 78,659; $\mathrm{gl}=15 ; \mathrm{p}=0,020$. El análisis factorial reveló la existencia de 4 com- ponentes que explican el $50,1 \%$ de la varianza (Tabla 9). Todos los ítems saturaron sobre 0,400 en algún factor. Esta sub-escala obtuvo un alfa de Cronbach de 0,289.

\section{Tabla 9}

Análisis factorial de los componentes principales después de rotación Varimax de la sub-escala Secuencial-Global.

\begin{tabular}{|c|c|c|c|c|}
\hline & Factor 1 & Factor 2 & Factor 3 & Factor 4 \\
\hline Pregunta 12 & 0,749 & & & \\
\hline Pregunta 20 & 0,544 & & & \\
\hline Pregunta 32 & 0,632 & & & \\
\hline Pregunta 24 & & 0,546 & & \\
\hline Pregunta 28 & & 0,651 & & \\
\hline Pregunta 36 & & $-0,667$ & & \\
\hline Pregunta 16 & & & 0,584 & \\
\hline Pregunta 44 & & & 0,725 & \\
\hline Pregunta 4 & & & & 0,441 \\
\hline Pregunta 8 & & & & 0,570 \\
\hline Pregunta 40 & & & & $-0,658$ \\
\hline$\%$ varianza & 14,449 & 12,667 & 12,057 & 10,982 \\
\hline$\%$ varianza total & & & & 50,155 \\
\hline
\end{tabular}

Factor 1 = definiendo contenidos; Factor 2 = razonando; Factor 3 = examinando.

En la Tabla 10 se observan las correlaciones entre las diferentes sub-escalas del Inventario de Felder-Silverman. Es posible observar que la escala activo-reflexivo se relaciona en forma con la escala visual-verbal $\left(\mathrm{r}=0,233 ; \mathrm{r}^{2}=0,05\right)$, la escala sensitivo-intuitivo se relaciona con la escala visual-verbal $\left(\mathrm{r}=0,167 ; \mathrm{r}^{2}=0,03\right)$ y la escala secuencial-global $\left(\mathrm{r}=0,229 ; \mathrm{r}^{2}=0,05\right)$. Finalmente, la escala visual-verbal se relaciona con la escala secuencial-global $\left(r=0,173 ; r^{2}=\right.$ $0,03)$.

Tabla 10

Correlaciones entre las diferentes sub-escalas del inventario de Felder-Silverman.

\begin{tabular}{lccc}
\hline & Activo-Reflexivo & Sensitivo-Intuitivo & Visual-Verbal \\
\hline Activo-Reflexivo & & & \\
\hline Sensitivo-Intuitivo & 0,032 & & \\
\hline Visual-Verbal & $0,233^{\star *}$ & $0,167^{\star}$ & \\
\hline Secuencial-Global & $-0,036$ & $0,229^{* *}$ & $0,173^{\star}$ \\
\hline
\end{tabular}

${ }^{\star}$ Correlación significativa al nivel 0,05

${ }^{* *}$ Correlación significativa al nivel 0,01 


\section{DISCUSIÓN}

Los resultados obtenidos en el presente estudio muestran índices de validación adecuados de cada una de las cuatro sub-escalas del Inventario de Kolb al ser aplicadas a estudiantes de educación física. Tres sub-escalas presentan 3 componentes que explican más del $60 \%$ de la varianza y una sub-escala presenta dos dimensiones con un $48 \%$ de varianza explicada. Sin embargo, los índices de confiabilidad obtenidos para cada escala a través del alfa de Cronbach están bajo el nivel de aceptabilidad, con valores de 0,200 y 0,400 .

En relación con el cuestionario de FelderSilverman, las cuatro sub-escalas mostraron índices de validez adecuados. Tres sub-escalas presentan 4 componentes que explican más del $50 \%$ de la varianza y una sub-escala con 5 componentes que explican el $60 \%$ de la varianza total. El índice de confiabilidad para cada escala también presenta valores bajo el nivel de aceptación.

El cuestionario de estilos de aprendizaje de Honey-Alonso abreviado (CHAEA-36) y el Inventario de dominancia cerebral de Herrmann presentan índices de validez y confiabilidad adecuados en estudiantes de educación física de Chile (Flores y Maureira, 2015, Maureira, 2015), razón por la cual resultan más aptos para evaluar a esta población.

\section{CONCLUSIÓN}

Se recomienda más estudios de las características del inventario de Kolb y del Cuestionario de Felder-Silverman en muestras de diversas universidades en las diferentes regiones del país, de esta forma sería posible confirmar las propiedades psicométricas de ambos instrumentos y así conocer si realmente se constituyen como una herramienta adecuada o no para evaluar los estilos de aprendizaje en esta población.

\section{REFERENCIAS BIBLIOGRÁFICAS}

Alonso, C., Gallego D. \& Honey, J. (1994). Los estilos de aprendizaje: procedimientos de diagnóstico y mejora. Bilbao: Ediciones Mensajero.

Al-Azawei, A., Parslow, P. \& Lundqvist, K. (2015) A psychometric analysis of reliability and validity of the index of learning styles (ILS). International Journal of Psychological Studies, 7(3), 46-57.

Brito, M. \& Espinosa, R. (2014). Evaluación de la fiabilidad del cuestionario sobre estilos de aprendizaje de Felder y Soloman en estudiantes de medicina. Investigación en Educación Médica, 4(13), 28-35.

Castaño, G. (2005). Independencia de los estilos de aprendizaje de las variables cognitivas y afectivo motivacionales. Tesis de Doctorado, Universidad Complutense de Madrid. Madrid, España.

Delgado, E. (2004). Relación entre los estilos de aprendizaje y estilos de pensamiento en estudiantes de maestría considerando las especialidades profesionales y el tipo de Universidad. Tesis doctoral, Facultad de Psicología, Universidad de San Marcos, Lima, Perú.

Dunn, K. \& Dunn, R. (1974). Learning style as a criterion for placement in alternative programs. Phi Delta Kappan 36, 275279.

Dunn, R. \& Dunn, K. (1978). Teaching students througth their individual learning styles: A practical approach. Reston, VA: Prentice Hall.

Escurra, L. (1992). Adaptación del inventario de estilos de aprendizaje de Kolb. Psicología, 9(1-2), 125-142.

Felder, R. \& Silverman, L. (1988). Learning and teaching styles in engineering education application. Engr Education, 78(7), 674-681. 
Flores, E. \& Maureira, F. (2015). Propiedades psicométricas del Inventario de Dominancia Cerebral en estudiantes de educación física. EmásF, Revista Digital de Educación Física, 6(36), 81-91.

Herrmann, N. (1990). The creative brain. Lake Lure: Brain Books.

Hosford, C. \& Siders, W. (2010). FelderSoloman's Index of Learning Styles: Internal consistency, temporal stability, and factor structure. Teaching and Learning in Medicine, 22, 298-303.

Hudak, M. \& Anderson, D. (1990). Formal operations and learning style predict success in statistics and computer science courses. Teaching of Psychology, $17,231-234$.

Kayes, D. (2005). Internal validity and reliability of Kolb's learning style inventory version 3 (1999). Journal of Business and Psychology, 20(2), 249-257.

Keefe, J. (1988). Aprendiendo perfiles de aprendizaje: manual de examinador. La Habana: Reston VA.
Kolb, D. (1984). Experiential learning: experience as the source of learning and development. New Jersey: Prentice Hall.

Maureira, F. (2013). Validez y confiabilidad del CHAEA en estudiantes de educación física de Chile. Educación Física Chile, 271, 8-15.

Maureira, F. (2015). CHAEA-36: adaptación del cuestionario Honey-Alonso de estilos de aprendizaje para estudiantes de educación física de Chile. Revista de Psicología Iztacala, 18(3), 1133-1152.

Puello, P., Fernández, D. \& Cabarcas, A. (2014). Herramienta para la detección de estilos de aprendizaje en estudiantes utilizando la plataforma Moodle. Formación Universitaria, 7(4), 15-24.

Willcoxson, L. \& Prosser, M. (1996). Kolb's Learning Style Inventory (1985): review and further study of validity and reliability. British Journal of Educational Psychology, 66(2), 247-257.

\section{Dirección para correspondencia}

Fernando Maureira Cid

PhD. en Educación. Docente Escuela de

Educación en Ciencias del Movimiento y

Deportes.

Universidad Católica Silva Henríquez.

Santiago de Chile

Contacto:

maureirafernando@yahoo.es

Recibido: 11/052017

Aceptado: 02/02/2018 